\title{
NOVOS HORIZONTES PARA A PRODUÇÃO CIENTÍFICA E TECNOLÓGICA
}

\author{
Fernanda Antônia da Fonseca Sobral ${ }^{*}$
}

\begin{abstract}
O presente artigo traça, inicialmente, uma visão panorâmica das novas tendências da produção do conhecimento. Nelas, destacam-se a importância assumida pela interação entre o conhecimento e a sociedade e outros aspectos como aplicabilidade e interdisciplinaridade do conhecimento, com a finalidade de contextualizar e descrever, nas suas principais características, alguns programas recentes de apoio à ciência e tecnologia (Fundos Setoriais, Institutos do Milênio e Institutos Nacionais de Ciência e Tecnologia). Finalmente, discutem-se alguns resultados de pesquisa realizada junto a pesquisadores de algumas áreas das Engenharias e da Sociologia, procurando apontar novos horizontes nas suas agendas de pesquisa.

PALAVRAS-CHAVE: conhecimento, política científica e tecnológica, inovação.
\end{abstract}

\section{INTRODUÇÃO}

A intenção deste artigo é dar, inicialmente, uma visão panorâmica dos novos caminhos da produção do conhecimento, com destaque para a importância assumida pela interação entre o conhecimento e a sociedade, com a finalidade de contextualizar e descrever, nas suas principais características, alguns programas recentes de apoio à ciência e tecnologia, sobretudo os Institutos Nacionais de Ciência e Tecnologia (INCTs), cabendo, finalmente, discutir alguns resultados de pesquisa realizada junto a pesquisadores de algumas áreas das Engenharias e da Sociologia, procurando apontar novos horizontes nas suas agendas de pesquisa.

\footnotetext{
* Doutorado em Sociologia. Professora do Programa de Pós- graduação em Sociologia da Universidade de Brasília (UnB). Pesquisadora do Centro de Gestão e Estudos Estratégicos (CGEE).

Campus Universitário Darcy Ribeiro - Asa Norte. Cep: 70910900. Brasília - Distrito Federal - Brasil. fernanda@unb.br
}

\section{NOVOS CAMINHOS NA PRODUÇÃO DO CONHECIMENTO}

Entre as condições socioinstitucionais que influenciam as políticas governamentais de ciência, tecnologia e inovação na atualidade e, consequentemente, a produção de conhecimento das universidades, podem-se destacar o processo de globalização, a democratização da sociedade e a revolução científica e tecnológica proporcionada pelas tecnologias da informação e comunicação (TICs).

Aglobalização aumenta a competitividade internacional e obriga as empresas a produzirem inovações tecnológicas, considerando o conhecimento especializado e de arranjos cooperativos com universidades, governo e outras empresas. Em decorrência, requer-se a dimensão econômica para a produção tecnológica. Além disso, a internacionalização da produção do conhecimento passa a ser cada vez mais uma necessidade.

Também o processo de democratização da sociedade faz com que, cada vez mais, a imprensa, as organizações não-governamentais (ONGs) e a 
sociedade civil organizada procurem exercer influência para que a produção científica e tecnológica tenha uma maior responsabilidade social. O próprio desenvolvimento dos meios de comunicação e informação possibilita a interação de diferentes atores e de diferentes instituições no processo de produção do conhecimento. Outrora contida essencialmente nos limites da comunidade acadêmica, a empreitada de produção e de apropriação do conhecimento tende a ampliar progressivamente os limites de seu universo em direção a um envolvimento maior de outros atores sociais.

Assim, segundo Velho (2010), a análise da produção do conhecimento se modifica passando de uma visão de ciência universalista, histórica e socialmente neutra, na qual a ideia de autonomia é proeminente, para uma concepção de ciência socialmente contextualizada.

Nesse contexto, Gibbons e seus colaboradores (1994) referem-se ao surgimento de um "novo modo de produção do conhecimento". Esse novo modo situa-se num contexto de aplicação, ou seja, são desenvolvidas pesquisas a partir da necessidade de resolver problemas práticos, e não apenas de interesses cognitivos, como na pesquisa básica. Também esse novo modo de produção do conhecimento é mais transdisciplinar do que disciplinar, pois, se o conhecimento é produzido no contexto de aplicação e não apenas com a intenção de acumulação do conhecimento na área, muitas vezes, o problema a ser solucionado por meio do conhecimento exige que disciplinas complementares trabalhem conjuntamente.

A partir das ideias de Gibbons (1994), Albert e Bernard (2002) realizaram estudos sobre a Economia e a Sociologia no Canadá, nos quais apontam para a definição de dois polos de produção de conhecimento: o polo PP (para pares), quando a legitimidade é conquistada por meio de uma produção destinada aos pares), e um polo PNP (para não-pares), cuja legitimidade é adquirida não apenas por meio de uma produção destinada aos pares, mas também voltada à demanda social de conhecimento e aberta à avaliação por outros atores sociais, não necessariamente pares.

Já Sobral e Trigueiro (1994), observando as tendências da produção do conhecimento, afirmam a emergência, no Brasil, não de um modelo único e exclusivo, mas de um modelo misto de desenvolvimento científico e tecnológico no qual estão associadas a pesquisa básica, a aplicada e tecnológica, a demanda espontânea à induzida, a comunidade científica a outros atores sociais, como o governo, as ONGs e o setor produtivo. ${ }^{1}$ Esse modelo procura unir a lógica do campo científico, ou seja, as demandas da própria evolução da ciência, às demandas econômicas e sociais, devendo reconhecer, porém, as diferenças entre áreas que apresentam necessidades distintas, umas mais aplicadas ou tecnológicas que outras, ou, ainda, umas mais articuladas às demandas das políticas públicas e das organizações não-governamentais e outras às demandas do setor produtivo.

Nessas concepções híbridas, segundo Nowotny (2006), não há incompatibilidade entre ciência real e ciência excelente, já que a ciência real surge e é construída pela ciência acadêmica e excelente. Assim, a ciência responde às várias pressões provenientes do Estado, da indústria e da sociedade e, de forma crescente, do mundo globalizado, sem diminuir a excelência, na medida em que há certo nível de autonomia, competição e seleção de propostas. Se a ciência real significar, igualmente, a ciência excelente, torna-se não apenas socialmente robusta, mas também cientificamente forte (Nowotny, 2006).

É o que também apontam estudos recentes, coordenados por Schwartzman (2008), sobre grupos de pesquisas de algumas universidades latino-americanas, conforme se pode observar na citação a seguir:

Apesar destas dificuldades, nossa pesquisa mostra que, em todos os países estudados, muitas equipes de pesquisa foram capazes de se abrir e fazer contribuições importantes para a sociedade, mantendo, ao mesmo tempo, a qualidade acadêmica do seu trabalho (2008).

${ }^{1}$ No caso da pesquisa biomédica e agrícola, desenvolvidas pela Fiocruz e pela Embrapa, essas tendências já são uma tradição. 


\section{PROGRAMAS DE APOIO À PESQUISA CIEN- TíFICA E TECNOLÓGICA}

No Brasil, atualmente, ao se analisar a política de ciência, tecnologia e inovação, constatamse novas fontes de financiamento, como os Fundos Setoriais, criados em 1999. Novos atores começam a participar do processo, com destaque para as empresas, e nova regulamentação foi implementada, tendo como foco o desenvolvimento da inovação. Já no final da década de 1990, começou a ser introduzida a inovação no discurso das políticas de C\&T.

Progressivamente, a inovação deixou de ser considerada apenas como resultado do processo de abertura do mercado e passou a ser objeto de uma série de medidas e de políticas ativas, tais como a criação dos Fundos Setoriais, o estabelecimento da Lei da Inovação, da Lei do Bem e da Política Industrial, Tecnológica e de Comércio Exterior (PICTE). Deve-se ressaltar que a preocupação com a inovação já estava também presente no Livro Branco do Ministério de Ciência e Tecnologia, de 2002, no qual se enfatiza a necessidade de

... criar um ambiente favorável à inovação que contribua para a competitividade das empresas e para o melhor aproveitamento da capacidade instalada em C\&T, de forma a acelerar os processos de transformação do conhecimento em serviços e produtos para a sociedade (MCT, 2002).

Podem-se observar essas tendências quando são considerados alguns programas de apoio à ciência, tecnologia e inovação do atual século. Constatase a preocupação com a relação entre conhecimento científico e tecnológico e a sociedade, na sua dimensão propriamente econômica da competitividade do setor produtivo, para a qual a inovação tecnológica é importante, e também na sua dimensão social, relacionada às políticas públicas e às tecnologias sociais. Constata-se também um estímulo ao avanço das fronteiras do conhecimento, ao lado do apoio à pesquisa aplicada e tecnológica, à constituição de projetos cooperativos entre as universidades e outras instituições públicas e privadas e à multidisciplinaridade e (ou) interdisciplinaridade.
Os Fundos Setoriais, por exemplo, foram concebidos como um instrumento novo de política científica e tecnológica no país, com relação a uma série de características. Uma delas é que, baseados nas teorias de inovação contemporâneas, os Fundos buscam mobilizar os vários agentes do processo inovativo, com destaque para as empresas. Outras características novas dos Fundos são: garantia da constância de recursos financeiros para o sistema de inovação, com foco em setores considerados estratégicos, e eficiência na gestão de tais recursos. Deve-se esclarecer que esses Fundos surgiram no bojo do processo de privatização de alguns setores da economia brasileira, como a exploração de petróleo, as telecomunicações e a energia elétrica, em decorrência da necessidade de garantir fontes públicas de recursos - relativamente estáveis - voltadas para o financiamento das ações de fomento da área de C\&T. Os recursos desses Fundos vêm de receitas fiscais extraorçamentárias, do faturamento das empresas atuantes em importantes setores da economia, que seriam utilizados para financiar projetos de pesquisa naqueles setores: petróleo, energia, transportes, telecomunicações, mineração, espaço, recursos hídricos, minerais, tecnologia da informação, biotecnologia, agronegócio, aeronáutico e saúde.

Em 2007, a Lei $\mathrm{n}^{0} 11.540 / 07$ regulamentou o Fundo Nacional de Desenvolvimento Científico e Tecnológico (FNDCT), incorporando, como suas fontes de financiamento, além da dotação de recursos orçamentários, todas aquelas que foram legalmente definidas como específicas para os Fundos Setoriais. Esse fato passou a garantir ao FNDCT recursos relativamente estáveis e independentes da disputa política quando da aprovação anual do Orçamento Geral da União. Também os Fundos têm ações transversais no sentido de que não necessariamente os recursos do Fundo Setorial de um setor financiam pesquisas ou desenvolvimento de tecnologias exclusivamente naquele mesmo setor, o que pode abrir certo espaço para pesquisas em temas sociais. Mas os editais referentes aos Fundos Setoriais são temáticos, tecnológicos ou aplicados e en- 
volvem grupos de pesquisadores. Na sua avaliação, é considerado o mérito, mas também as possibilidades de inovação tecnológica em cooperação com o setor produtivo e, em menor grau, o desenvolvimento de tecnologias sociais, como, por exemplo, tecnologias para comunidades tradicionais e para pequenos produtores rurais.

Já o programa Institutos do Milênio, criado em 2001, tinha como objetivo principal aumentar a competência científica brasileira em áreas importantes da ciência e da tecnologia, mediante o apoio e a capacitação de centros de pesquisa e laboratórios de alta qualidade científica e (ou) atuantes em áreas estratégicas para o desenvolvimento do país. No documento básico do programa, percebem-se claramente as tendências apontadas, tais como a abordagem temática do fomento, que supõe um tratamento multi e interdisciplinar para o tratamento das questões propostas, quer em sua concepção organizacional, que deve prever novos arranjos institucionais capazes de superar as tradicionais divisões acadêmicas entre disciplinas ou áreas do conhecimento, quer no tocante à formação de parcerias entre o setor público $\mathrm{e}$ o privado e à articulação de redes de competências deâmbito nacional e internacional.

A inovação deve ser a marca dos Institutos do Milênio, seja no estabelecimento de novas técnicas nas atividades de pesquisa básica ou na previsão de efetivos mecanismos de transferência constituir de institutos atuantes em áreas estratégicas, definidas segundo as prioridades do Programa de Ciência e Tecnologia do MCT. Em 2001, tinha como meta financiar um projeto para cada uma das seguintes áreas: Amazônia, Semiárido e Recursos do Mar. De fato, foram aprovados apenas dois institutos dessa categoria, o Instituto do Milênio do Semiárido: Biodiversidade, Bioprospecção e Conservação de Recursos Naturais; e o Instituto do Milênio de Oceanografia: Uso e Apropriação de Recursos Costeiros. Na demanda espontânea, foram aprovados 15 Institutos do Milênio.

Em 2005, foram aprovados 34 Institutos do Milênio, dos quais cinco constituem uma continuidade daqueles aprovados em 2001, sendo 14 da demanda induzida e 20 da demanda espontânea, podendo ser agrupados em alguns temas ou áreas, tais como: Ciências Exatas e Tecnologia da Informação (8), Saúde (8), Biotecnologia e Nanotecnologia (7), Meio Ambiente eBiodiversidade (5) e seis institutos cujas temáticas se aproximam mais das Ciências Humanas. As novidades trazidas pelo segundo edital foram o maior número de institutos aprovados na demanda induzida e a inclusão de temas propriamente sociais, como Violência e Segurança Pública, Estratificação Social e Desigualdade e Democracia e Cidadania, Observatório das Metrópoles, não abrangidos nos primeiros Institutos do Milênio de 2001.

O novo programa, constituído pelos INCTs, teve seus projetos aprovados ao final de 2008. Segundo o documento de orientação aprovado pelo seu comitê de coordenação, os Institutos do Milênio estabeleceram-se como poderoso instrumento para fazer avançar a ciência e tecnologia no país, mas o Ministério da Ciência e Tecnologia (MCT) se propõe agora a dar nova dimensão à iniciativa, por meio do programa dos Institutos Nacionais, que tem metas mais ambiciosas e abrangentes em termos nacionais, destacando-se:

- Mobilizar e agregar, de forma articulada, com atuação em redes, os melhores grupos de pesquisa em áreas de fronteira da ciência e em áreas estratégicas para o desenvolvimento sustentável do país, como definidas no Plano de Ação Ciência, Tecnologia e 
Inovação parao Desenvolvimento Nacional(PACTT).

- Impulsionar a pesquisa científica básica e fundamental competitiva internacionalmente.

- Desenvolver pesquisa científica e tecnológica de ponta, associada a aplicações, promovendo a inovação e o espírito empreendedor, em estreita articulação com empresas inovadoras, nas áreas do Sistema Brasileiro de Tecnologia (Sibratec).

- Promover o avanço da competência nacional em sua área de atuação, criando, para tanto, ambientes atraentes e estimulantes para alunos talentosos de diversos níveis, do ensino médio ao pós-graduado, e responsabilizando-se diretamente pela formação de jovens pesquisadores. Os Institutos Nacionais devem ainda estabelecer programas que contribuam para a melhoria do ensino de ciências e com a difusão da ciência para o cidadão comum.

- Apoiar a instalação e o funcionamento de laboratórios em instituições de ensino e pesquisa e empresas, em temas de fronteira da ciência e da tecnologia, promovendo a competitividade internacional do país, a melhor distribuição nacional da pesquisa científico-tecnológica e a qualificação do país em áreas prioritárias para o seu desenvolvimento regional e nacional (BRASIL, 2008).

Assim, as missões principais dos INCTs devem ser a promoção da pesquisa de vanguarda e de elevada qualidade em temas de fronteira e (ou) estratégicos, a formação de recursos humanos e a transferência de conhecimento para a sociedade, o que pressupõe um programa de educação e difusão de conhecimento focalizado no fortalecimento do ensino médio e na educação científica da população. Esse aspecto significa um estímulo à utilização de outros instrumentos para divulgação dos resultados das pesquisas, além da publicação científica, ou seja, à produção para não-pares (PNP). Para aqueles INCTs voltados para a aplicabilidade do conhecimento, também é requerida a transferência do conhecimento para o

${ }^{2}$ Deve-se observar que a transferência do conhecimento para a sociedade é restrita a ações de divulgação e educação científica, não incluindo a transferência do conhecimento para o setor produtivo e para o governo, considerada como outra missão daqueles INCTs que realizam pesquisa aplicada. setor empresarial ou para o governo. ${ }^{2} \mathrm{~A}$ intenção é desenvolver a cadeia de conhecimento, ou seja, desde a elaboração das ideias e conceitos até produtos comerciais ou contribuições para a formulação de políticas públicas, que é a situação mais próxima das Ciências Humanas e Sociais.

O Documento de Orientação dos INCTs previa a aprovação de 45 INCTs, cerca de 20 na demanda espontânea, que poderia incluir qualquer área de conhecimento, e cerca de 25 em temas induzidos, que tivessem relação direta com as áreas estratégicas do Plano de Ação em CT\&I. Desses últimos, faziam parte, entre outros, alguns temas mais próximos das Ciências Humanas, tais como Inclusão Social, Segurança Pública, Defesa Nacional, Educação, Saúde. ${ }^{3}$ Porém, ao final, foram aprovados 123 INCTs, contando com maior volume de recursos - cerca de 605 milhões de reais - provenientes de várias fontes, como FNDCT, Fundações de Amparo à Pesquisa (FAPs), Conselho Nacional de Desenvolvimento Científico e Tecnológico (CNPq), Coordenação de Aperfeiçoamento de Pessoal de Nível Superior (Capes), Banco Nacional de Desenvolvimento Econômico e Social (BNDES), Petrobras e Ministério da Saúde.

Atualmente, estão em funcionamento 120 INCTs (considerando as demandas espontânea e induzida) nas seguintes áreas de conhecimento e (ou) temas : Saúde, que teve o maior número de projetos contemplados (36), Ecologia e Meio Ambiente, (16) Agrárias (14), Engenharia e TIC (13), Humanas e Sociais (11) Energia(10) , Exatas e Naturais (10) e Nanotecnologia (10) .

Deve-se observar que alguns dos Institutos do Milênio se transformaram em INCTs. A demanda induzida teve cerca de $60 \%$ de aprovação em relação à demanda espontânea, o que já indica uma tendência de a pesquisa estar se orientando por algumas prioridades estratégicas.

${ }^{3}$ Além desses, os outros temas da demanda induzida foram: Biotecnologia, Nanotecnologia, Tecnologias da Informação e Comunicação, Biocombustíveis, Energia Elétrica, Hidrogênio e Fontes Renováveis de Energia, Petróleo, Gás e Carvão Mineral, Agronegócio, Biodiversidade e Recursos Naturais, Amazônia, Semiárido, Mudanças Climáticas, Programa Espacial, Programa Nuclear, Mar e Antártica. 
A área considerada como Ciências Humanas e Sociais aborda os seguintes temas:

- INCT de Estudos das Metrópoles;

- INCT de Observatório das Metrópoles;

- INCT de Políticas Públicas, Estratégias e Desenvolvimento;

- INCT de Educação, Desenvolvimento Econômico e Inserção Social;

- INCT de Estudos Comparados em Administração Institucional de Conflitos;

- INCT sobre Violência, Democracia e Segurança Cidadã:

- INCT de Comportamento, Cognição e Ensino;

- INCT para Estudos sobre os Estados Unidos;

- INCT Brasil Plural;

- INCT de Inclusão no Ensino Superior e na Pesquisa;

- INCT de Psiquiatria do Desenvolvimento para Crianças e Adolescentes (INPD).

No seminário de avaliação dos INCTs,${ }^{4}$ pôde-se perceber claramente, nessa experiência, a associação entre a pesquisa básica e a aplicada, já com algumas contribuições para a formulação de políticas públicas (sobretudo no caso da política de segurança pública, da política urbana e mesmo das políticas de quotas no ensino superior e outras políticas para minorias), ou mesmo de tecnologias de intervenção social nos campos de segurança pública e acesso à justiça. Também começaram a se preocupar com outras formas de divulgação da ciência (com utilização intensiva das novas tecnologias da informação e comunicação), além das publicações científicas, inclusive com algumas ações voltadas para o ensino médio, como é o caso do INCT de Comportamento, Cognição e Ensino, com destaque para o uso de tecnologias da informação nos processos de aprendizagem de crianças com dificuldades e (ou) deficiências congênitas. Ademais, observaram-se contribuições com outras formas de educação, como é o caso do INCT sobre Violência, Democracia e Segurança Cidadã, que tem, entre as suas atividades, a capacitação

${ }^{4}$ Nesse seminário, realizado em novembro de 2010 , em Brasília, acompanhei os relatos do grupo de INCTs Ciências Humanas e Sociais como representante do CGEE, que tem a responsabilidade de avaliar o programa INCTs, cabendo ao CNPq a avaliação dos projetos individuais. de policiais, e do INCT Brasil Plural, que colabora com organizações indígenas para a educação indígena.

Ao mesmo tempo, trabalha-se para avançar nas contribuições teóricas, no conhecimento de vanguarda e na internacionalização das redes de pesquisa e do próprio conhecimento. No que concerne à interdisciplinaridade, quando acontece, ela se dá pela participação de pesquisadores de diferentes áreas de conhecimento, mas geralmente da mesma grande área de Ciências Humanas, com algumas exceções, como é o caso do INCT de Observatório das Metrópoles e do INCT de Políticas Públicas, Estratégias e Desenvolvimento. Mesmo assim, reúnem-se pesquisadores das Ciências Humanas e das Ciências Sociais Aplicadas, que têm grande proximidade. ${ }^{5}$ Quanto à organização em redes de produção do conhecimento, embora ainda haja INCTs concentrados no mesmo estado e na mesma região, constata-se, nesse grupo de INCTs, a participação de 369 pesquisadores de 61 diferentes instituições, embora mais de $50 \%$ das instituições e pesquisadores sejam do Sudeste, depois do Sul (19\%) e do Nordeste (15\%), proporções aproximadas àquelas já previstas no documento de orientação para a distribuição regional dos recursos - cerca de $50 \%$ para o Sudeste, $15 \%$ para o Sul e 35\% para as regiões Norte, Nordeste e Centro-Oeste - dada a grande participação da Fundação de Amparo à Pesquisa do Estado de São Paulo (Fapesp) como financiadora. ${ }^{6}$ Ou seja, embora as desigualdades regionais ainda permaneçam, é relevante destacar algumas cooperações que estão ocorrendo entre pesquisadores de diferentes localidades por meio do estabelecimento das redes de pesquisa. Mas deve-se observar também que, dentre as instituições, além de universidades no país e no exterior, também participam organizações nãogovernamentais e secretarias municipais, como no caso do INCT de Observatório das Metrópoles.

\footnotetext{
Excecão é o INCT de Psiquiatria do Desenvolvimento para Crianças e Adolescentes (INPD), cujos pesquisadores são predominantemente da área de Saúde.

${ }^{6}$ Esses dados foram fornecidos pelo CNPq e cabe observar que a demanda total aprovada de INCTs teve a participação de aproximadamente $60 \%$ de propostas e de pesquisadores do Sudeste.
} 


\section{NOVOS HORIZONTES NAS AGENDAS DE PESQUISA}

Essas novas tendências apontadas pelos programas de apoio à ciência, tecnologia e inovação já estão afetando a produção científica e tecnológica nas universidades e a percepção dos pesquisadores sobre $\mathrm{o}$ atendimento, por meio das suas pesquisas, de certas demandas do ambiente externo à universidade?

Alguns resultados podem ser analisados em uma pesquisa intitulada "Demandas Sociais e Lideranças Científicas"”, que tinha como objetivo analisar a produção científica e tecnológica nas Engenharias e na Sociologia e, ao mesmo tempo, a política científica e tecnológica dos governos FHC Lula, a fim de verificar em que medida outras demandas sociais, além das demandas do próprio conhecimento, e outros atores sociais, além dos próprios pesquisadores, estão influenciando a política científica e tecnológica e a produção de conhecimento.

Foram escolhidas disciplinas com perfis diferentes, como Engenharia Mecânica, Engenharia Elétrica e Engenharia Florestal, que têm um perfil mais aplicado e tecnológico com tendência a se voltar para as demandas do setor produtivo, e a Sociologia, com uma tendência teórico-empírica, mas que pode retraduzir demandas das políticas públicas e de organizações não-governamentais.

Os pesquisadores $1 \mathrm{~A}$ e 2 do CNPq foram identificados pela Plataforma Lattes nas Engenharias (Elétrica, Mecânica e Florestal) e na Sociologia, a fim de verificar se a posição no campo científico leva a posições diferentes no que se refere a essa questão. Após essa etapa, elaborou-se um questionário eletrônico sobre uma série de aspectos, entre os quais: razões da escolha do objeto de pesquisa, instituições parceiras, fontes de financiamento, ocorrência ou não de demandas provenientes de instituições externas à universidade, como também a autopercepção do pesquisador sobre a sua produção. Esse questionário ele${ }^{7}$ Nessa pesquisa, contei com a colaboração, em diferentes fases, da então doutoranda e hoje doutora Tatiana Maranhão e dos bolsistas PIBIC Izabela Amaral Caixeta Krislane de Andrade Matias, Natalia Peres Kornijezuk, Paulo Victor Silva Pacheco e Roberto Rego Mendes. trônico enviado a todos os pesquisadores dessas áreas, situados nos níveis citados, totalizou um universo de 472 pesquisadores em 2008. Obtiveram-se respostas de 122 questionários, sendo que $71 \%$ dos respondentes são provenientes das áreas de Engenharias. A Engenharia Elétrica teve mais expressividade quanto ao retorno dos questionários, com 33\% de respostas, seguida da Sociologia, com 29\%, e da Engenharia Mecânica, com $28 \%$, e, por último, pela Engenharia Florestal, com baixa proporção de respostas, isto é, com $11 \%$. Isso se coaduna com o fato de que o universo de pesquisadores analisados da Engenharia Elétrica conta com o maior número de pesquisadores (144).

As principais razões para a escolha do objeto de pesquisa pelos respondentes na área de Sociologia, em ambos os níveis, concentra-se no interesse teórico. No nível 1A, em segundo lugar das razões mais citadas, observa-se a influência de obra científica relevante, com 25\%. Já no nível 2, a segunda razão mais apontada foi a influência de movimentos sociais ao lado da influência de obra científica relevante com $16 \%$. Desse modo, a perspectiva acadêmica e teórica da Sociologia é muito importante em ambos os níveis, porém, no nível 2 , outras influências começam a aparecer.

Nas três Engenharias, também a principal razão para a escolha do objeto de estudo é o interesse teórico. No que se refere especificamente à Engenharia Elétrica, o interesse teórico foi apontado por 33\% das respostas dos pesquisadores de ambos o níveis (1A e 2), e a demanda de empresas obteve a mesma proporção entre os pesquisadores 1A (33\%), ainda que, no nível 2, essa opção tenha sido escolhida por apenas $11 \%$. Outra diferença para o nível 2 é que $20 \%$ apontaram a influência do orientador, razão não-indicada pelos pesquisadores de nível mais alto, já que esses pesquisadores já se titularam há muito tempo. Isso sugere que as Engenharias já estão trabalhando associadamente ao setor produtivo e que os pesquisadores $1 \mathrm{~A}$, com maior reconhecimento científico, estão apresentando maior potencial para desenvolver trabalhos cooperativos com empresas. 
Gráfico 1 - Razões de escolha do objeto de pesquisa na Sociologia

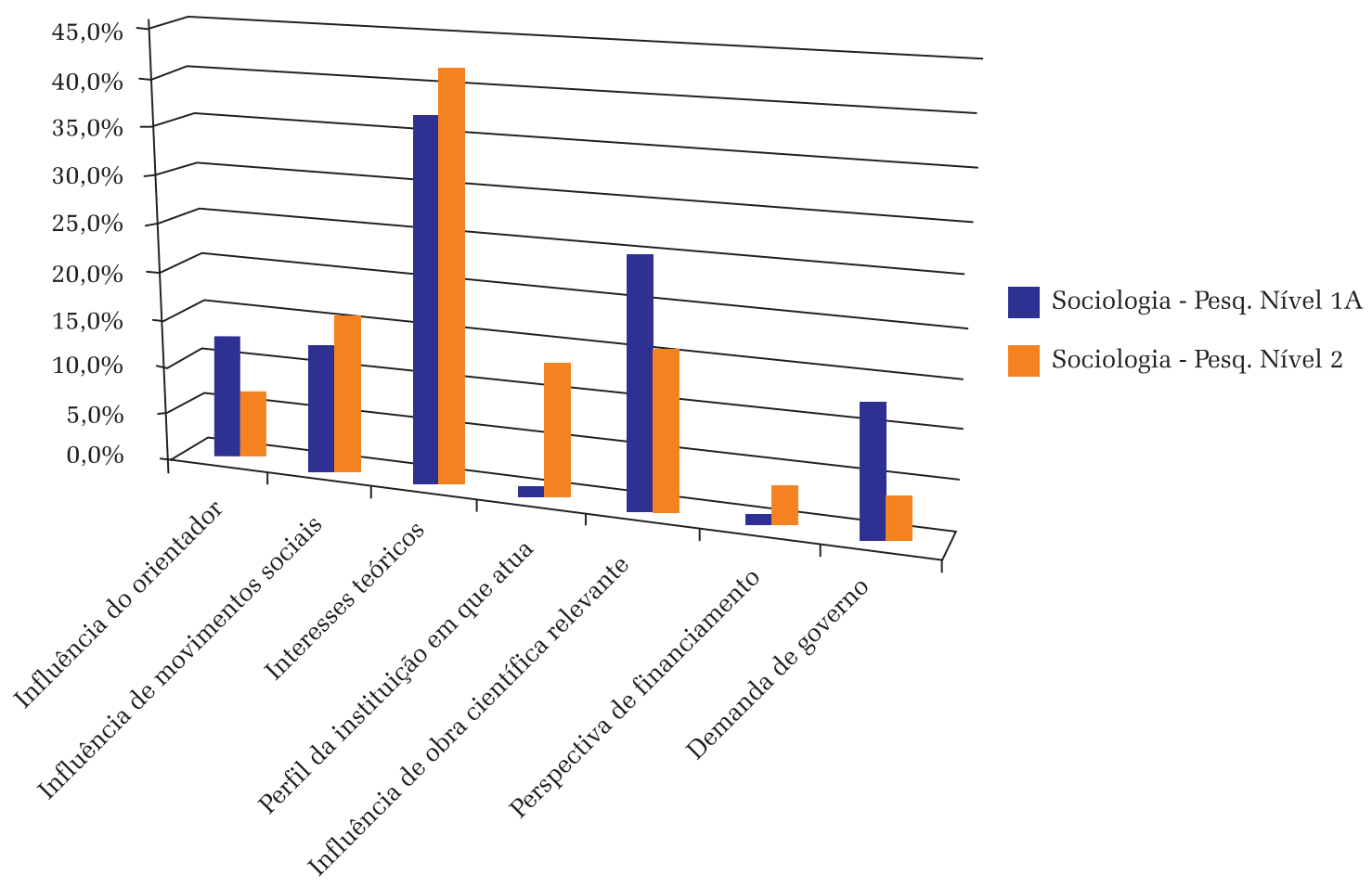

Em relação à Engenharia Florestal, o interesse teórico e a demanda de empresas foi a resposta predominante entre os pesquisadores 2 , obtendo aproximadamente $30 \%$ de respostas. Talvez por ser uma área relativamente nova dentro das Engenharias e relacionada à preservação ambiental, seus pesquisadores são também influenciados na escolha do objeto de pesquisa, ainda que em proporção reduzida, por outros fatores, como os movimentos sociais (cerca de $10 \%$ entre os pesquisadores nível 2), um dado que não aparece nas outras Engenharias.

Na Engenharia Mecânica, o interesse teórico como razão principal da escolha de objeto de pesquisa teve 36\% de respostas no nível 1A e 22\% no nível 2. A razão perspectiva de financiamento obteve $29 \%$ de respostas no nível 1A. A demanda de empresa, na Engenharia Mecânica, foi indicada por apenas $7 \%$ dos pesquisadores $1 \mathrm{~A}$ e por $14 \%$ no nível 2. Em resumo, nas Engenharias, de um modo geral, as pesquisas se dão principalmente por interesse teórico e por demandas empresariais (em menor proporção na Engenharia Mecâni- ca), indicando que há uma associação entre pesquisa básica, aplicada e desenvolvimento tecnológico, entre o interesse propriamente científico e a possibilidade de aplicação.

No que concerne à parceria com outras instituições na realização dos projetos de pesquisa, ao considerar o total de respostas da Sociologia, a participação mais frequente ( $57 \%$ entre os pesquisadores $1 \mathrm{~A}$ e $28 \%$ entre os pesquisadores 2) é de órgãos do governo federal (ministérios, agências reguladoras), evidenciando a importância desse conhecimento para as políticas públicas. Nota-se, também, que, entre os pesquisadores do nível 2 da Sociologia, existe uma maior variedade de instituições parceiras exteriores à universidade, que participam dos projetos, já que buscaram não só as instituições governamentais ou reguladoras, mais frequentes entre os pesquisadores $1 \mathrm{~A}$, mas também procuraram o apoio de ONGs, organismos internacionais e órgãos do governo estadual ou municipal, ainda que numa proporção pequena.

Nas Engenharias, verifica-se, sobretudo, a participação das empresas de grande porte e de ór- 
Gráfico 2 - Razóes de escolha do objeto de pesquisa na Engenharia Florestal, Mecânica e Elétrica

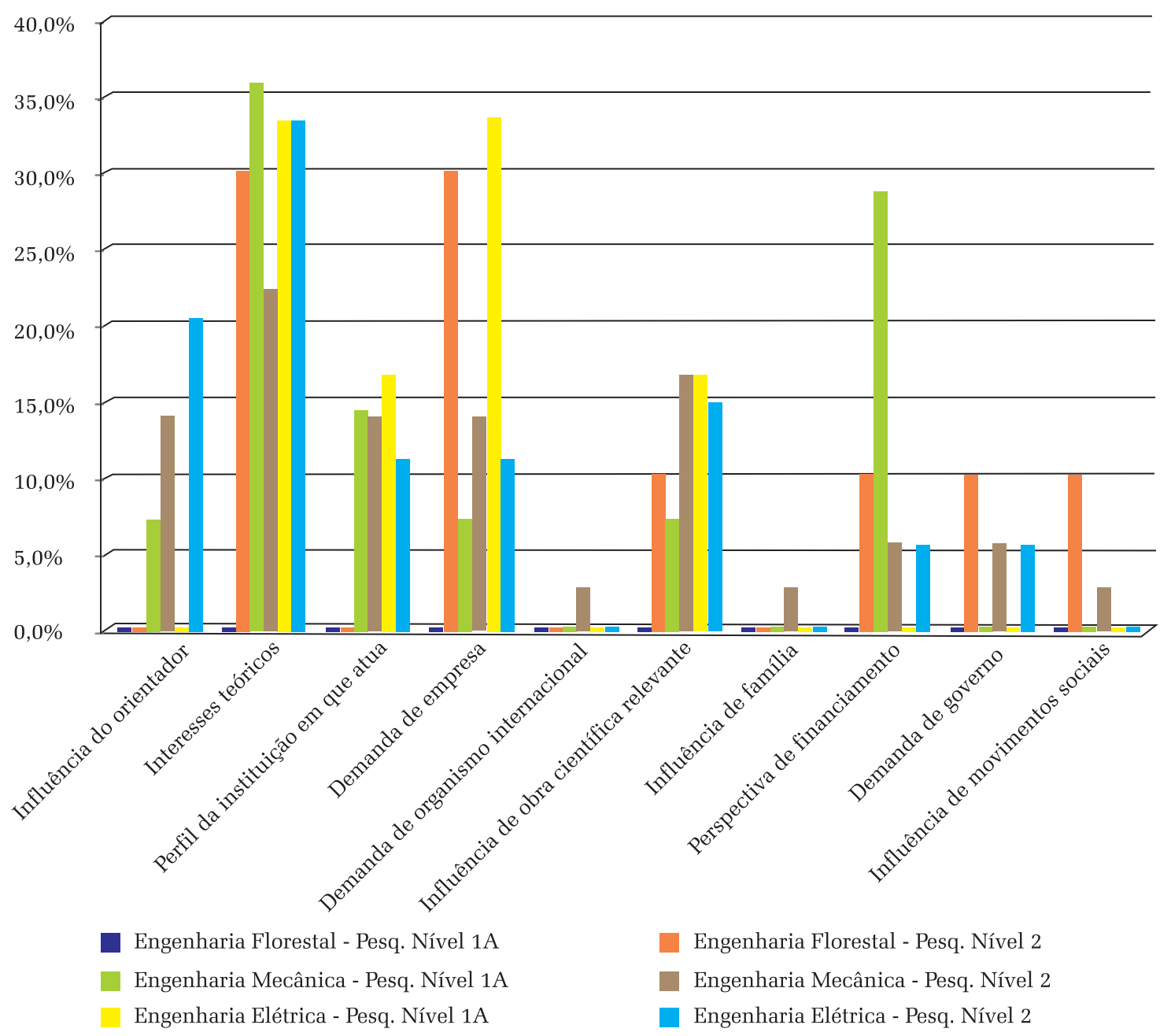

gãos do governo federal (ministérios, agências re- tes dos projetos realizados, em proporções míniguladoras) na realização dos projetos de pesquisa, mas, enquanto no nível 1A essa tendência não é com exceção da Engenharia Florestal, cuja partici- constatada.

pação das ONGs foi indicada por $47 \%$ das respostas, dado o fato de que as florestas são tratadas por várias instituições desse tipo, sendo que a participação das grandes empresas e dos órgãos federais foi indicada por apenas $13 \%$ das respostas. Já na Engenharia Mecânica, as grandes empresas e os órgãos federais foram indicados pela mesma proporção (27\%) e, na Elétrica, por $26 \%$ quanto aos órgãos federais e por $22 \%$ no que se refere a grandes empresas. Porém vale ressaltar que, na Engenharia Florestal, no nível 2, aparecem também órgãos do governo estadual e(ou) municipal (secretarias) e empresas de médio porte como participan-

Em relação às fontes de financiamento de pesquisa dos respondentes, as agências de fomento nacional (CNPq, Capes) constituem a principal fonte de financiamento, tanto no nível 1A quanto no nível 2 da área de Sociologia. A segunda opção, nos dois níveis, coube às Fundações de Apoio à Pesquisa, com proporção maior de respostas entre os pesquisadores $1 \mathrm{~A}$.

Também as principais fontes de financiamento na Engenharia Elétrica são as agências de fomento nacionais (CNPq, Capes) e as FAPs com $29 \% \%$. Em seguida, aparecem as empresas de grande porte (em torno de 20\%), e órgãos do governo 
Gráfico 3 - Fontes de financiamento dos projetos de pesquisa na Sociologia

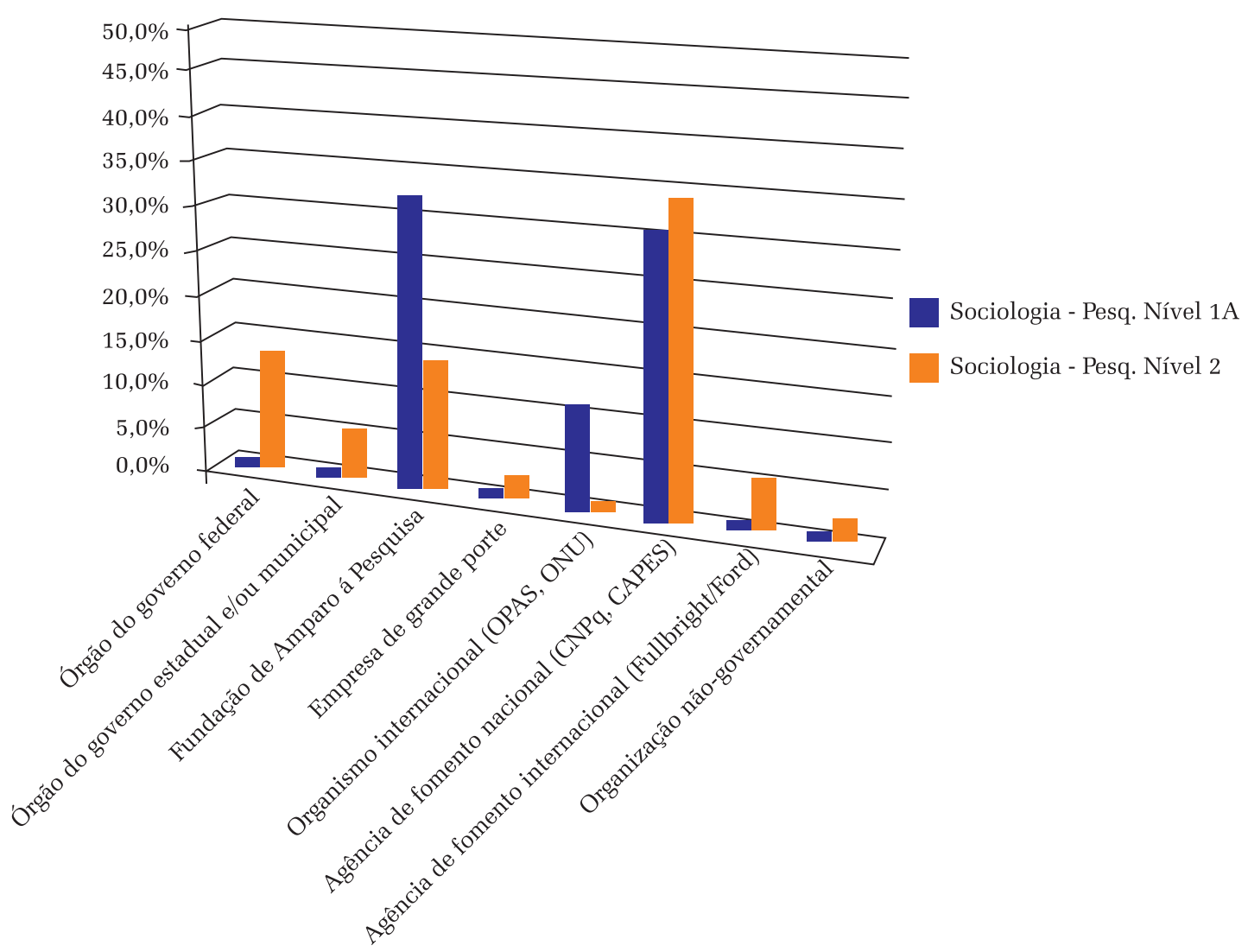

federal (ministérios, agências reguladoras). Essa ordem, ainda que com proporções um pouco diferentes, constata-se tanto entre os pesquisadores 1A como naqueles de nível 2.

Na área de Engenharia Mecânica, o tipo de

financiamento que obteve mais respostas foi a das empresas de grande porte, porém as outras opções referentes às FAPs e às agências de fomento nacionais (CNPq, Capes) tiveram proporções muito próximas. No nível 1A, a principal fonte de financiamento indicada foi a empresa de grande porte, com $32 \%$ das respostas, diferentemente dos pesquisadores do nível 2, em que essa mesma fonte de financiamento obteve $22 \%$ das respostas e cuja principal fonte de financiamento apontada se refere a órgãos do governo federal, com $27 \%$ das respostas. É importante observar que, embora na Engenharia Mecânica não tenham sido enfatizadas as demandas do setor produtivo como razão para escolha do objeto de estudo, as empresas são parceiras de alguns projetos e também possibilitam financiamento.
Na área de Engenharia Florestal, a principal fonte de financiamento apontada pelos respondentes foram os órgãos do governo federal (ministérios, agências reguladoras), em torno de $30 \%$. Em seguida, as empresas de grande porte, as FAPs, e as agências de fomento nacional (CNPq, Capes), essas últimas citadas em proporção reduzida pelos respondentes.

Por fim, buscou-se verificar, por meio da autopercepção de cada pesquisador sobre a sua produção, a partir de dados coletados no Diretório de Grupos de Pesquisa/CNPq, a contribuição da produção de cada respondente a partir de três dimensões sugeridas: avanço/desenvolvimento da ciência, inovação tecnológica e inovação social/ solução de problemas sociais, ilustradas nos gráficos 5 e 6.

A maioria de respostas na Sociologia, de uma forma geral, apontou a contribuição da sua produção para o avanço/desenvolvimento da ciência. Em relação às diferenças encontradas nos dois 


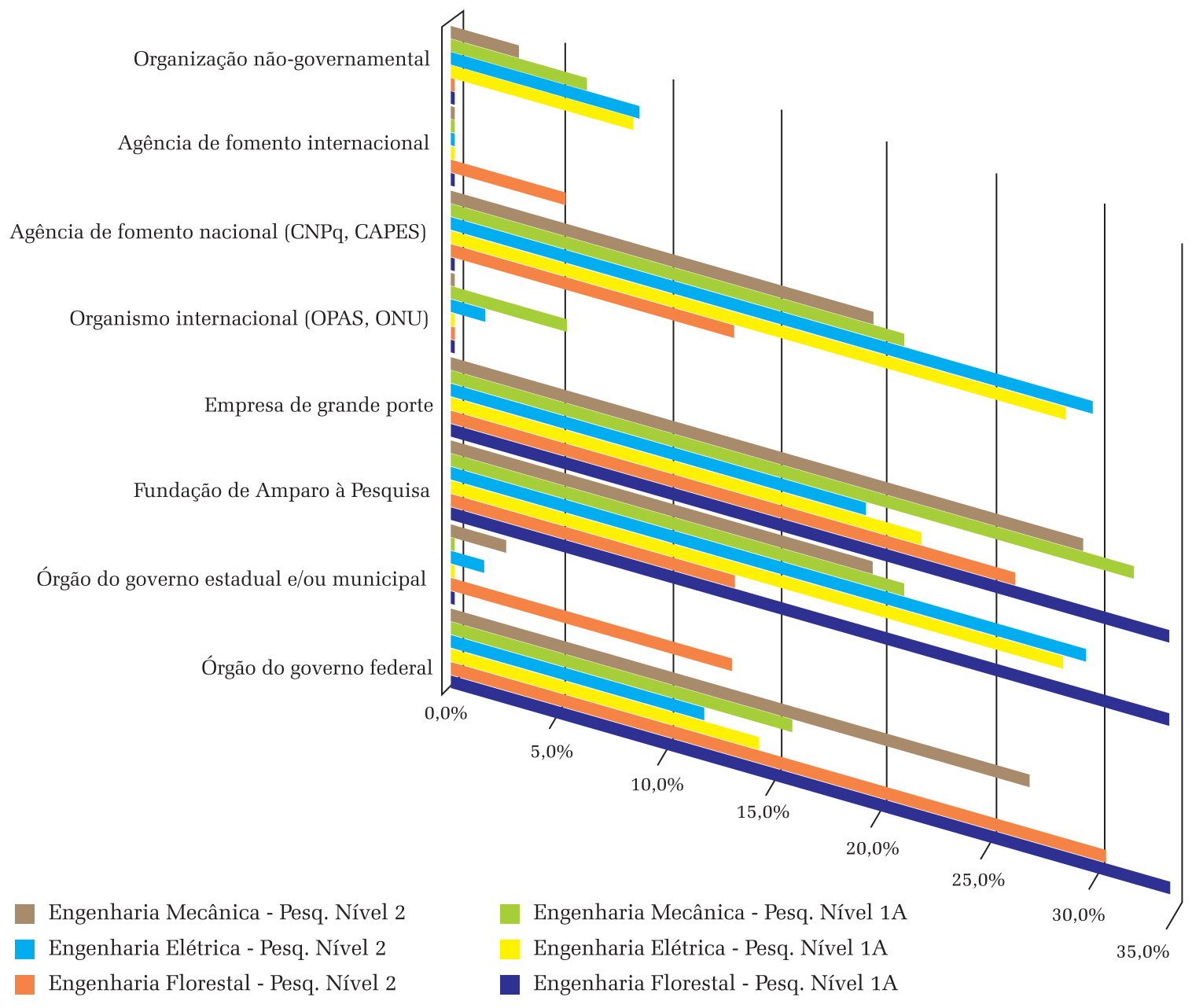

níveis da Sociologia, percebe-se que os pesquisadores $1 \mathrm{~A}$ deram apenas como resposta o avanço/ desenvolvimento da ciência, expressando um perfil bem acadêmico da produção. No nível 2 da Sociologia, o item avanço/desenvolvimento da ciência também foi a opção mais frequente, com $58 \%$. Em seguida, aparece o item inovação social/solução de problemas sociais, com $33 \%$ das respostas. Essa diferença de percepção sobre a produção entre os dois níveis revela coerência das suas respostas com as anteriores, no que se refere às razões de escolha do objeto de estudo e às parcerias nas atividades de pesquisas.

Na Engenharia Elétrica, 50\% das respostas dos pesquisadores $1 \mathrm{~A}$ e $40 \%$ dos pesquisadores 2 responderam que sua produção contribui para a inovação tecnológica, e, ao contrário, 40\% e 50\% das respostas dos pesquisadores $1 \mathrm{~A}$ e 2 , respectivamente, apontaram o avanço/desenvolvimento da ciência. Por último, mas surpreendentemente, a contribuição para a inovação social/solução de problemas sociais, foi indicada por $10 \%$ das respostas. As diferenças entre os dois níveis são insignificantes nessa questão. Um pesquisador nível 2, da Elétrica, chegou a mencionar acerca da inovação social existente em seu trabalho:

O produto que estamos desenvolvendo, modem PLC para acesso residencial à rede de dados, é de baixo custo e contribuirá para diminuir a exclusão digital no país e no mundo. Além disso, estamos formando mão de obra qualificada na área de tecnologia na região da zona da mata mineira. 


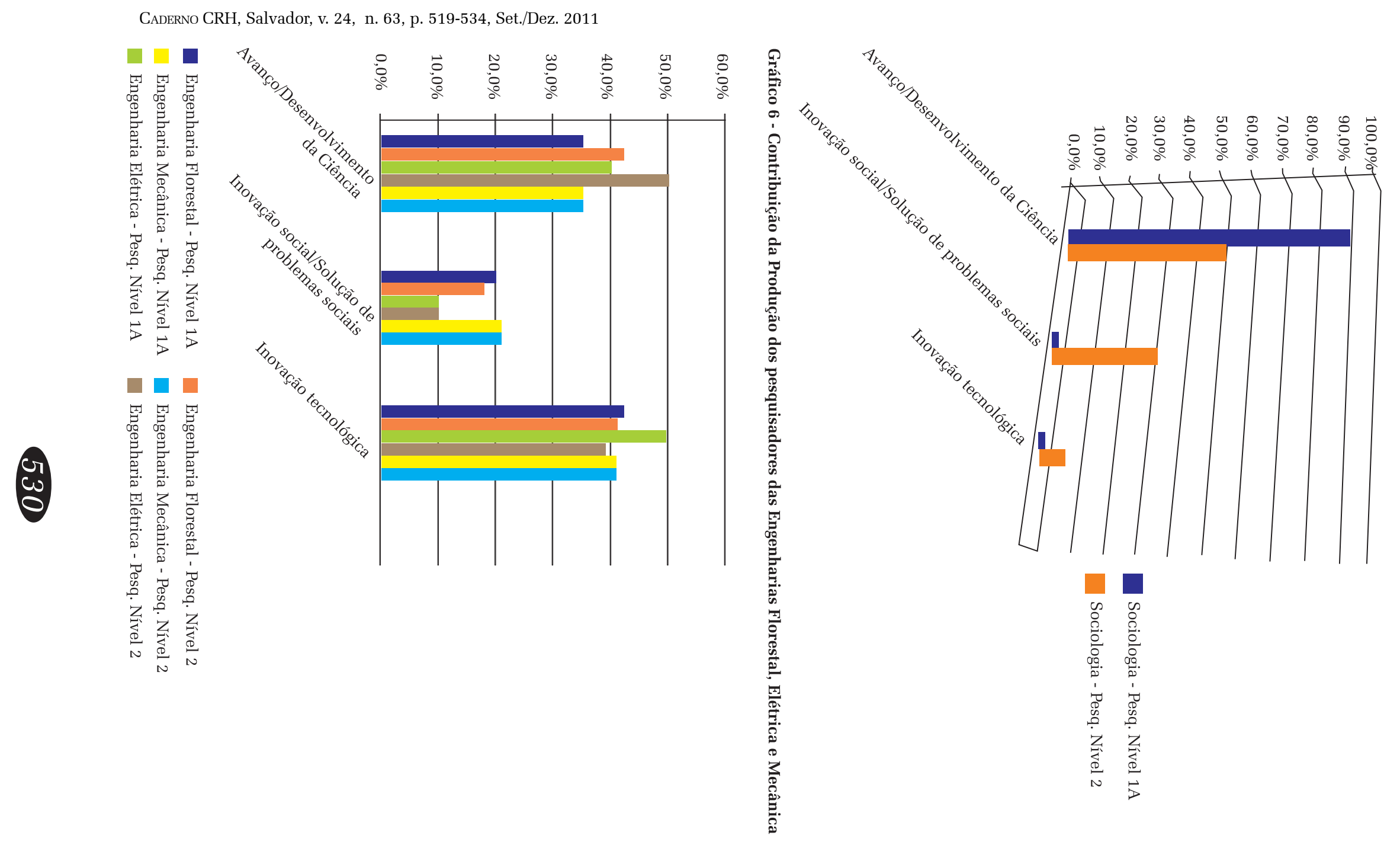


Entre os pesquisadores $1 \mathrm{~A}$ e 2 da Engenharia Mecânica, a resposta mais frequente foi a sua contribuição para inovação tecnológica, com $43 \%$, seguida pelo avanço/desenvolvimento da ciência, com $35 \%$. Já a contribuição para a inovação social/solução de problemas sociais foi a menos expressiva entre os pesquisadores de ambos os níveis, mas obtendo $22 \%$ das respostas dos pesquisadores 1A e 2 de Engenharia Mecânica. Quanto à dimensão de inovação social, foi citado por um pesquisador nível 1A o seguinte:

Provavelmente, minha maior contribuição foi propiciar que estudantes de Engenharia criassem uma empresa de alta tecnologia em simulação de complexos problemas industriais, que hoje tem mais de 100 empregados, sendo 12\% doutores, $37 \%$ mestres, $22 \%$ cientistas da computação e 29\% engenheiros. É uma empresa que oferece empregos para doutores, uma realidade que se busca há muito no Brasil. Todo o pessoal técnico é de nível superior. [...] Com a criação de empresas de alta tecnologia contribui-se para o aprimoramento da tecnologia disponível para as empresas nacionais, geram-se empregos. Nosso relacionamento com o setor produtivo e atualmente, principalmente Petrobras, contribui socialmente porque estamos ajudando a melhorar a tecnologia disponível para as empresas e com isso a geração de empregos.

A contribuição da produção dos pesquisadores da Engenharia Florestal para inovação tecnológica foi a principal dimensão apontada pelos pesquisadores 1A e 2, com $45 \%$ e $42 \%$. Em seguida, vem o avanço/desenvolvimento da ciência com $35 \%$ e $42 \%$, e, por ultimo, a inovação social/ solução de problemas sociais, com 20\% e 16\%.

É interessante notar a importância da contribuição para o avanço da ciência e, ao mesmo tempo, para a inovação tecnológica na produção dos pesquisadores das diferentes Engenharias analisadas e nos diferentes níveis. Deve-se também observar que a inovação social aparece, embora em proporções reduzidas, na percepção dos pesquisadores das Engenharias, seja pela criação de empresas e de empregos, seja pela geração de produtos de baixo custo.

\section{CONSIDERAÇÕES FINAIS}

Se, ao longo dos últimos governos, a análise dos programas Fundos Setoriais, Institutos do Milênio e INCTs aponta para um estímulo à pesquisa tecnológica e aplicada associada à pesquisa básica, a projetos cooperativos com outras instituições exteriores à universidade e à interdisciplinaridade, observa-se, por outro lado, que essas tendências estão sendo incorporadas pelos pesquisadores gradativamente, talvez em maior grau pelas pesquisas que estão apoiadas por programas específicos, como os INCTs, refletindo-se, ainda de forma embrionária, na produção de conhecimento e com diferenças a partir das tradições e culturas disciplinares.

Os pesquisadores, além de se referirem ao interesse teórico e à sua contribuição para o avanço do conhecimento, também já indicam outras influências externas e as suas contribuições para a inovação social ou solução de problemas sociais, principalmente na Sociologia, e sua contribuição para a inovação tecnológica, sobretudo nas Engenharias, evidenciando, mais uma vez, a associação entre pesquisa básica, aplicada e desenvolvimento tecnológico.

Começam também a ser estabelecidas algumas parcerias com empresas, com governo e com organizações não-governamentais, ainda que em pequena escala. Mas as parcerias se dão, sobretudo, a partir de contatos pessoais com pesquisadores, evidenciando uma frágil institucionalização da interação entre universidade e sociedade.

Outra dimensão que se destaca é a diferença entre os perfis das disciplinas e, ao mesmo tempo, algumas diferenças entre os níveis de pesquisadores, apontando que o reconhecimento acadêmico dos pesquisadores $1 \mathrm{~A}$ pode facilitar a obtenção de apoios externos para as suas pesquisas, provenientes do governo e do setor produtivo, o que mostra a compatibilidade entre a ciência real e a ciência excelente (Nowotny, 2006).

Ainda que as empresas já financiem alguns estudos, sobretudo nas Engenharias, as principais fontes de financiamento continuam sendo as agên- 
cias de fomento e as FAPs. Porém o fato de os editais de fomento estarem se voltando cada vez mais para o financiamento de temas estratégicos, numa abordagem interdisciplinar e com tendência à aplicabilidade, e o fato de o conhecimento gerado pelas Engenharias ser considerado mais aplicável do que o conhecimento gerado pela Sociologia torna as Engenharias mais conhecidas pelos não-pares e mais inclinadas para a chamada produção para não-pares (PNP).

Deve-se acrescentar que, numa análise dos editais de fomento, nos quais todos os pesquisadores $1 \mathrm{~A}$ e 2 das áreas selecionadas (não apenas os respondentes ao questionário) tiveram projetos aprovados, constata-se uma predominância de financiamento, nas Engenharias, em editais vinculados aos Fundos Setoriais, ao passo que, na Sociologia, os financiamentos das pesquisas se dão, sobretudo, por meio dos editais universais, abertos a todas as áreas de conhecimento e de editais específicos para as Ciências Humanas e Sociais.

O conhecimento produzido pela Sociologia é considerado mais teórico e, portanto, mais restrito aos espaços acadêmicos (PP). Sendo assim, há uma demanda maior por instituições externas ao mundo acadêmico nas Engenharias do que na Sociologia, como é o caso do governo e das empresas nas três Engenharias analisadas e das ONGs na Florestal. Porém, no caso da Sociologia, começam a

... as diferenças entre áreas com necessidades distintas, umas mais aplicadas ou tecnológicas que outras, ou ainda, umas mais articuladas às demandas das políticas públicas e das organizações nãogovernamentais e outras às demandas do setor produtivo (Sobral; Almeida; Caixeta, 2008).

Pode-se afirmar, então, que a tendência, na Sociologia, é oferecer um conhecimento que retraduz ou reflete demandas da sociedade, com certo nível de "autonomia reflexiva" (Maranhão e Sobral, 2008), e que pode subsidiar políticas públicas, ao passo que, nas Engenharias, essa de- manda se faz cada vez mais frequente e direta por parte do governo e do setor produtivo na orientação dos rumos da produção do conhecimento.

Se, no século passado, a ciência falou para a sociedade, neste século, a sociedade passa a falar para a ciência (Nowotny; Scott; Gibbons, 2001). Os ruídos da sociedade começaram a ter eco, de formas diferenciadas, apontando novos horizontes para a pesquisa, mas há ainda um vasto caminho a ser trilhado quanto à institucionalização dessa interação.

(Recebido para publicação em 05 de agosto de 2011) (Aceito em 07 de dezembro de 2011)

\section{REFERÊNCIAS}

ALBERT, M.; BERNARD, P. Faire utile ou faire savant? La nouvelle production de connaissances et la sociologie universitaire québecoise. ISociologie et Sociétés, Montreal, Université de Montreal, v.32, n.1, p.71-92, 2002.

ASSOCIACÃO BRASILEIRA DE DESENVOLVIMENTO INDUSTRIAL (ABDI). Talentos para inovação: engenharias, física, matemática, química. Brasília, 2007. (Documento provisório)

BALBACHEVSKY, Elizabeth. Processos Decisórios em política científica, tecnológica e de inovação no Brasil. nova geração da política de ciência, tecnologia e inovação. Brasília: CGEE-MCT, 2010.

Recursos humanos em áreas estratégicas para a inovaçã̃o: a experiência internacional. Formação de recursos humanos em áreas estratégicas de ciência, tecnologia e inovação. Brasília: CGEE-MCT, 2010.

BRASIL. Ministério da Ciência e Tecnologia. Plano Plurianual do Ministério da Ciência e Tecnologia. Brasília, 2000.
Plano Estratégico. Brasília, 2004.
inovaçã̃o plano de ação de ciência, tecnologia e
idesenvolvimento nacional. Brasília: 2007.

. Plano Estratégico. Brasília, 2004.
inovaçã̃o para o desenvolvimento nacional. Brasília: 2007. MCT/CNPq. Programa Institutos do Milênio. Brasília: 2001. Documento básico

Programa Institutos Nacionais de C\&T. Brasília: 2 2008. Documento de orientação

GIBBONS, M. et al. The new production of knowledge: the dynamics of science in contemporany societies. London: Sage, 1994

GROENEWEGEN, P. Accommodating science to external demands: the emergence of Dutch toxicology. Science, Technology \& Human Values, London, v.4, n.27, p.479-498, 2002.

NOWOTNY, H. Real science is excellent science: how to interpret post academic science, mode 2 and the ERC. 
Journal of Science Communication, London, v.5, n. 4, p.234259, dec. 2006.

; SCOTT, P.; GIBBONS, M. Re-Thinking science. Cambridge: Polity Press, 2001.

SCHWARTZMAN, S. Pesquisa universitária e inovação no Brasil. In: AVALIAĈ̃̃O de políticas de ciência, tecnologia e inovação: diálogo entre experiências internacionais e brasileiras. Brasília: CGEE, 2008.

. As universidades latinoamericanas e sua contribuição para o desenvolvimento sustentável da região. Rio de Janeiro: Biblioteca Virtual de Ciências Humanas, 2008.

SOBRAL, F.A.F.; TRIGUEIRO, M.G.S. Limites e potencialidades da base técnico-científica. In: FERNANDES, A.M; SOBRAL, F.A.F. (Org.) Colapso da ciência e da tecnologia no Brasil. Rio de Janeiro: RelumeDumará, 1994.

A universidade e o novo modo de produção do conhecimento Caderno CRH: Centro de Recursos Humanos da UFBA, Salvador, n.34, p.265-275, jan./jun. 2001.

; ALMEIDA, M.R.C.; CAIXETA, M.V.C. As lideranças científicas. Ciências \& Cognição, Rio de Janeiro, v.13, p.179-191, 2008.

; MARANHÃO, Tatiana de P.A La autonomia reflexiva de la sociologia. Íconos, Quito, v.31, p.140-158, 2008.

VELHO, L.M.S. Modos de produção de conhecimento e inovação. Estado da arte e implicações para a política científica, tecnológica e de inovação. Brasília: CGEE, 2010. 


\section{NEW HORIZONS TO SCIENTIFIC AND TECHNOLOGICAL PRODUCTION}

\author{
Fernanda Antônia da Fonseca Sobral
}

This present article draws, initially a overview of the new tendencies for production of knowledge. On them stands out the importance taken by the interaction between, knowledge, the society and other aspects such as applicability and interdisciplinary of the knowledge, with the aim to contextualize and describe, on their main characteristics, some recent programs to support sciences and technology ( Sectoral Funds, Institute of Millennium and National Institute of Sciences and Technology). Finally some results from researches done with the aid of researchers from some fields of Engineering and Sociology were discussed, seeking to point out new horizons in their research agenda.

KEY WorDs: knowledge, technological and scientific policy, innovation.

\section{NOUVEAUX HORIZONS POUR LA PRODUCTION SCIENTIFIQUE ET TECHNOLOGIQUE}

\author{
Fernanda Antônia da Fonseca Sobral
}

Cet article donne tout d'abord une vision panoramique des nouvelles tendances de la production de la connaissance. On y remarque tout particulièrement l'importance de l'interaction entre la connaissance et la société ainsi que d'autres aspects tels que la convenabilité et l'interdisciplinarité de la connaissance. L'objectif est de mettre en contexte et de décrire, dans leurs grandes lignes, quelques programmes récents de soutien à la science et à la technologie (Fundos Setoriais, Institutos do Milênio e Institutos Nacionais de Ciência e Tecnologia/Fonds Sectoriaux, Instituts du Millénaire et Instituts Nationaux de Science et Technologie). Finalement quelques résultats d'une enquête faite auprès de chercheurs du domaine de l'Ingénierie et de la Sociologie sont présentés afin de signaler de nouveaux horizons dans leurs programmes de recherche.

Mots-CLÉs: connaissance, politique scientifique et technologique, innovation.

Fernanda Antônia da Fonseca Sobral - Doutora em Sociologia. Pós-doutoramento em Paris na École das Hautes Études em Sciences Sociales. Professora do Programa de Pós- graduação em Sociologia da Universidade de Brasília (UnB). Pesquisadora colaboradora senior da Universidade de Brasília e pesquisadora visitante no CGEE (Centro de Gestão e Estudos Estratégicos). Tem experiência na área de Sociologia com ênfase em Teoria Sociológica, atuando principalmente nos seguintes temas: educação, tecnologia, ciencia, universidade e pesquisa. Publicação mais recente: Dilemas e questões para o futuro das Ciências Sociais no desenvolvimenro científico e tecnológico atual. In: Ribeiro,G.L; Fernandes, A.M; Martins, C.B; Filho, W.T. (Org.). As ciências sociais no mundo contemporâneo. Brasilia: Editora da UNB, 2011, p. 1-308. 